\title{
Dorsal Psammomatous Meningioma Revealed by Sciatica Case Report
}

\section{Daouda Wague ${ }^{1}$, Maguatte Gaye², Cheikh Ndiaye Sy3 ${ }^{3}$ Mouhamet Cisse ${ }^{4}$, Souleymane Diallo5, Youssouf Sakho ${ }^{6}$}

\author{
${ }^{1}$ Neurosurgeon at CHMFT, Touba, Senegal \\ ${ }^{2}$ Professor of Neurosurgery (UCAD) and Head of Neurosurgery Unit at HOGIP, Dakar, Senegal \\ ${ }^{3}$ Neurosurgeon and Senior Researcher at UCAD, Dakar, Senegal \\ ${ }^{4}$ Resident in Neurosurgery at FANN, Touba, Senegal \\ ${ }^{5}$ Resident in Neurosurgery at HOGIP, Dakar, Senegal \\ ${ }^{6}$ Professor of Neurosurgery at UCAD, Dakar, Senegal \\ Email: wagued@yahoo.fr, magou762003@yahoo.fr, cheikh-sy@live.fr, mohabcisse@gmail.com, souldiallo307@gmail.com, \\ yousakho@yahoo.fr
}

How to cite this paper: Wague, D., Gaye, M., Sy, C.N., Cisse, M., Diallo, S. and Sakho, Y. (2021) Dorsal Psammomatous Meningioma Revealed by Sciatica Case Report. Open Journal of Orthopedics, 11, 164-169. https://doi.org/10.4236/ojo.2021.115016

Received: April 8, 2021

Accepted: May 25, 2021

Published: May 28, 2021

Copyright $\odot 2021$ by author(s) and Scientific Research Publishing Inc. This work is licensed under the Creative Commons Attribution International License (CC BY 4.0)

http://creativecommons.org/licenses/by/4.0/

\section{(c) (i) Open Access}

\begin{abstract}
Introduction: Meningiomas are benign tumors most localized in brain. Intraspinal meningiomas are rare and may have an intra or extradural topography. We report the case of a dorsal psammomatous meningioma revealed by sciatica. Case description: We present a 58-year-old female hospitalized for bilateral painful and disabling L5 sciatica rebellious to medical treatment and physiotherapy. At physical examination, she presented a lumbar spinal syndrome without root deficit. MRI (Magnetic Resonance Imaging) showed a borderline lumbar canal with a paramedian L4-L5 herniated disc. She undergone a first surgery a L4 decompressive laminectomy and there was no postoperative complications. The follow-up was marked by partial pain regression. Three months later, after a fall she presented low back pain and progressive worsening gait troubles. A MRI was realized and shown L3-L4, L4-L5 duct narrowing. A second surgery a L3 and L5 decompressive laminectomy was performed. The postoperative follow up was characterized by the worsening of the motor deficit to $2 / 5$ associated with brisk osteotendinous reflexes. A medullary MRI was realized, showing an intradural but extra-medullary compressive lesion facing D1-D2. The indication of tumor removal was made and complete resection was performed. She totally recovered from the deficit and the pain. The pathology report showed a psammomatous meningioma. Conclusion: This case report emphasizes the atypical clinical presentation of spinal meningiomas and the importance of a meticulous neurological examination to avoid misdiagnosis.
\end{abstract}




\section{Keywords}

Meningioma, Dorsal, Sciatica, Psammomatous

\section{Introduction}

Spinal cord tumors are classified in extradural or intradural. Intradural tumors are divided into intramedullary or extramedullary. The most common extramedullary tumors are schwannomas, neurofibromas and meningiomas. Intraspinal meningiomas are generally benigns tumors that develop from proliferation of arachnoid cells. These are rare tumors of the spinal level and mainly are intradural but their localizations can be sometimes extradural and in this case they are most often confused with metastatic neoplasms [1].

Spinal meningioma represents approximately $30 \%$ of extramedullary intradural tumors of the spine and $8 \%$ of all meningiomas [2]. Pain was the predominant symptom, often antedating other symptoms by months or years (paresthesias, cold sensations, hot sensations, numbness, weakness, bowel or bladder dysfunction, chest pain). Sensory loss and weakness were the next most common symptoms, followed by bladder and bowel dysfunction [3].

The prognosis depends on the quality of the resection. If the diagnosis of meningioma is evident, complete resection should be attempted to reduce the risk of recurrence.

We report the case of a 58-year-old female diagnosed of dorsal meningioma which was revealed by the recurrence of an operated lombosciatica.

\section{Case Description}

We report the case of a 58-year-old female received in our hospital for disabling bilateral L5 low back pain rebellious to medical treatment and physiotherapy. On examination, she presented segmental stiffness with fingertips to floor distance of $5 \mathrm{~cm}$. There was no radicular sensitivo-motor deficit or sphincter disorders. MRI revealed a limit lumbar canal with an L4-L5 paramedian herniated disc in conflict with the L5 roots (Figure 1). The indication for an L4-L5 laminectomy was made. Intraoperatively, only an L4 laminectomy was performed because the disc was flat and the roots free. Postoperatively, there was a clinical improvement marked by pain regression.

Three months later, after a fall, she presented low back pain with a poorly systematized radiculalgia and progressive worsening gait troubles evolving into relative functional impotence. At physical examination, the patient had a motor deficit of the two lower limbs estimated 3/5 and sphincters disorders. The follow-up MRI showed an L3-L4, L4-L5 duct narrowing. The indication of L3-L5 laminectomy was made and performed. The postoperative follow up were marked by the worsening of the motor deficit to $2 / 5$ associated with brisk osteotendinous reflexes. A medullary MRI was realized, showing a hypointense intradural and 
extra-medullary lesion in T2 compressing the spinal cord facing D1-D2 (Figure 2). The indication of tumor removal was made. During surgery tumor was found to be intradural and extramedullary after laminectomy of D1, D2 and D3. Complete resection of the lesion was performed.

The evolution was marked by motor recovery with muscle strength rated $4 / 5$ in the lower limbs. After 2 months of postoperative follow-up, the patient walks with a walker. The pathology reports showed a psammomatous meningioma.

\section{Discussion}

Spinal meningiomas usually are rare benigns tumors which develop from proliferation of arachnoid cells; most often solitary. They grow slowly and most often affect the female. Intraspinal meningiomas are mainly intradural. They represent approximately $25 \%$ of primary spinal cord tumors [4] [5] [6].

An analysis of intradural spinal meningiomas reported in the literature revealed a mean age of occurrence around 50 years with a clear female predominance [3] [7]. It has been suggested that spinal meningioma occurs more frequently in fertile women, because of the possible dependency of these tumors on

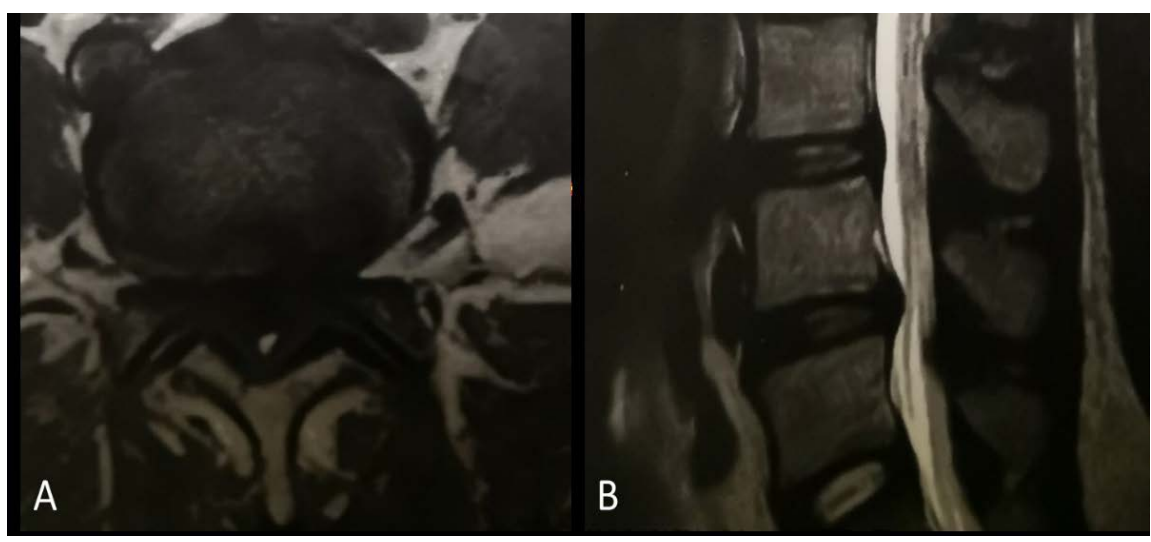

Figure 1. MRI of the lumbar spine T2 séquence, in axial (A) et Sagital (B) section showing a limit lumbar canal with posteromedial disc protrusion in L4L5.

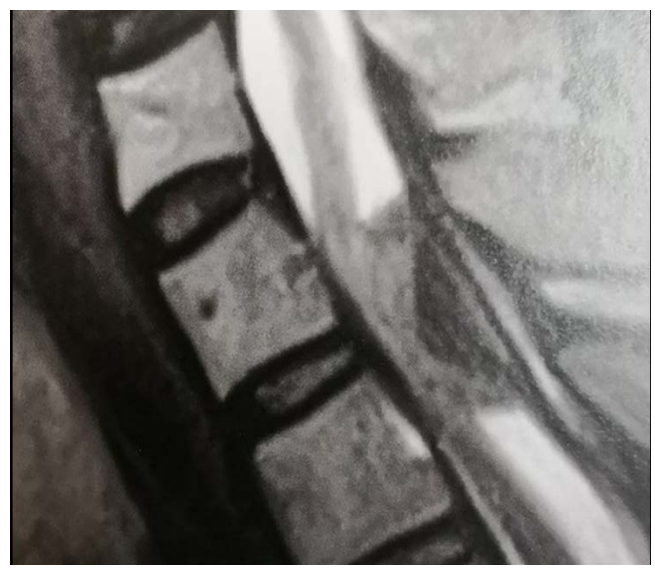

Figure 2. MRI of the dorsal spine T2 sequence in sagital section showing a hypointense intradural extramedullary lesion compressing spinal cord in D1-D2. 
sex steroid hormones [8]. Although the theory about the effect of hormones on meningioma has been controversial, hormonal studies have shown the existence of various receptor types (steroid, peptidergic, growth factor, and aminergic) in meningioma [9]. They are characterized by slow growth; which explains the progressive appearance of clinical signs. These long unrecognized signs may evolve to simple radicular damage to spinal cord compression syndrome. Pain is the most common symptom, especially when it is isolated, it can often misdiagnose. Radicular, funicular, or localized back pain was the predominant symptoms. Significant weakness (paresis or plegia) and sensory loss (hypoesthesia, paresthesia, or anesthesia) were the next most common symptoms. Therefore, this tumor has been confused with multiple sclerosis, syringomyelia, pernicious anemia, and herniated disc [8].

Some authors have reported that correct treatment was delayed by misdiagnosis in $24 \%$ of cases [10]. Others report $32 \%$ of diagnostic error [3]. In this case report we showed the misdiagnosis which led to lumbar canal decompression surgery due to sciatica. The persistence of sciatica after surgery, the worsening of the motor deficit and the existence of pyramidal signs such as brisk osteotendinous reflexes allowed us to make the diagnosis on spinal cord MRI.

Magnetic resonance imaging is the best imaging technique for diagnosis [8]. It allows a better analysis of the medullary tissue, in all plans. The spine is explored in T1, T2 weighted sequence and after intravenous injection of gadolinium, which allows differentiation between intramedullary tumors and extramedullary or extradural intradural tumors. The MRI specifies the localization of the lesion, and the characterization of the extramedullary intradural lesions which can simulate a spinal meningioma. Meningiomas appear as isosignal in relation to the cord in T1 and T2 weighted sequences, if they are calcified, they appear as hypointense in $\mathrm{T} 1$ and $\mathrm{T} 2$. The injection of gadolinium enhances the meningioma in a homogeneous and intense manner on the $\mathrm{T} 1$ and $\mathrm{T} 2$ weighted sequences. In our case, MRI revealed an intradural and extra-medullary lesion in compressive T2 hypointense on the marrow facing D1-D2. The most frequent location of spinal meningioma is the thoracic region in the intradural-extramedullary space [11].

Effective treatment for meningiomas is undoubtedly surgery, the aim is to relieve spinal cord compression while minimizing any trauma to the spinal cord and its vascularization. The principles of treatment are based on a complete resection of the meningioma and its dura-mater base to avoid recurrence. Dissection need the using of microscope and tumor removal is facilitated by the use of careful low-flow aspiration and bipolar coagulation.

The anatomopathological results showed a psammomatous meningioma in our case, different to the literature where the meningothelial form is the most frequent [3] [12].

Physiotherapy is an important part of neurological deficits treatment. It improves the results of surgical treatment and increases the chances of neurological 
recovery [13]. The degree of neurological recovery is very high in intraspinal meningiomas, those in better neurological condition preoperatively obtain better results. However long term results show that even paraplegics are not in desperate situations [3]. In our case, the evolution after surgery was good with motor recovery, muscle strength increased from 2 to 4 after two months of follow-up.

\section{Conclusion}

This case report emphasizes atypical clinical presentation of spinal meningiomas and shows the value of examining well the patients, in this case a complete and meticulous neurological examination could help to avoid misdiagnosis. If there is a doubt, perform a complete MRI of the spine to avoid missing a hidden lesion.

\section{Agree to Conditions}

All authors of the manuscript have read and agreed to its content and are accountable for all aspects of the accuracy and integrity of the manuscript.

\section{Patient Consent}

The patient consent to participate to this study and allow us to publish the case

\section{Conflicts of Interest}

The authors declare no conflicts of interest regarding the publication of this paper.

\section{References}

[1] McPhee, S.J., Papadakis, M.A., Tierney, L.M. Jr., Gonzales, R. and Zeiger, R. (2007) Current Medical Diagnosis \& Treatment. 46th Edition, The McGraw-Hill Companies, New York, 20

[2] Westwick, H.J and Shamji, M.F. (2015) Effects of Sex on the Incidence and Prognosis of Spinal Meningiomas: A Surveillance, Epidemiology, and End Results study. Journal of Neurosurgery: Spine; 23, 368-373. https://doi.org/10.3171/2014.12.SPINE14974

[3] Levy, W.J. Jr., Bay, J. and Dohn, D. (1982) Spinal Cord Meningioma. Journal of Neurosurgery, 57, 804-812. https://doi.org/10.3171/jns.1982.57.6.0804

[4] Heiskanen, O. (1968) Benign Extramedullary Tumors in the High Cervical Region. Annales Chirurgiae et Gynaecologiae Fenniae, 57, 59-62.

[5] Kernohan, J.W. and Sayre, G.P. (1952) Tumors of the Central Nervous System. Atlas of Tumor Pathology, Section X, Fascicle 35. Armed Forces Institute of Pathology, Washington DC, 141.

[6] Sloof, J.L., Kernohan, J.W. and MacCarty, C.S. (1964) Primary Intramedullary Tumors of the Spinal Cord and Filum Terminale. WB Saunders, Philadelphia/London, 165-166.

[7] Rand, C.W. (1963) Surgical Experiences with Spinal Cord Tumors. A Survey over A Forty-Year Period. Bulletin of the Los Angeles Neurological Society, 28, 260-268.

[8] Ferruh, G., Serdar, K., Zafer, C., et al. (2000) Review of 36 Cases of Spinal Cord Me- 
ningioma. Spine, 25, 727-731.

[9] Nelson, J.S., Parisi, J.E. and Schochet, S.S. (1993) Principles and Practice of Neuropathology. In: Parisi, J.E., Mena, H., Eds., Non-Glial Tumors, Mosby-Year Book, St. Louis, 203-213

[10] Davis, R.A. and Washburn, P.L. (1970) Spinal Cord Meningiomas. Surgery, Gynecology and Obstetrics, 131, 15-21.

[11] Solero, C.L., Fornari, M., Giombini, S., et al. (1989) Spinal Meningiomas: Review of 174 Operated Cases. Neurosurgery, 125, 153-160.

[12] Kabre, A., Sakoyo, Y. and Gueye, M. (1992) Intra-Spinal Meningiomas Based on 14 Cases. Dakar Medical, 37, 2.

[13] Calmels, P., Fayolle, I., Valayer, E. and Bethoux, F. (2000) Rehabilitation of Spinal Cord Injuries. Medical Competition, 122, 678-682.

\section{Abreviations}

MRI: Magnetic Resonance Imaging 